\title{
Reversible predictors of reversion from mild cognitive impairment to normal cognition: a 4-year longitudinal study
}

\author{
Hiroyuki Shimada ${ }^{{ }^{*}}$ D, Takehiko Doi ${ }^{1}$, Sangyoon Lee ${ }^{1}$ and Hyuma Makizako²
}

\begin{abstract}
Background: Although previous studies have revealed many factors related to mild cognitive impairment (MCl) reversion, information about reversible factors related $\mathrm{MCl}$ reversion is limited, impeding the development of intervention strategies. The aim of the present study was to examine whether reversible factors such as lifestyle activities are associated with $\mathrm{MCl}$ reversion in elderly individuals using the National Center for Geriatrics and Gerontology_Study of Geriatric Syndromes database. A total of 396 community-living older adults (age $\geq 65$ years) participated in the study. They were classified as reverters or non-reverters from mild cognitive impairment to normal cognition. We assessed lifestyle activities, potential confounding factors of cognitive decline, and reversion of mild cognitive impairment.

Results: In a completed data set of 396 participants, 202 participants (51.0\%) reverted from MCI to normal cognition. The reversion rate in participants for whom we imputed data was 34.3\%. In the imputed group, a logistic regression model showed that the odds ratios (ORs) for reversion were significantly higher in participants who drove a car (OR 1.50, 95\% confidence interval (Cl) 1.41-1.60), used a map to travel to unfamiliar places (OR 1.12, 95\% Cl 1.06-1.18), read books or newspapers (OR 1.54, 95\% Cl 1.37-1.73), took cultural classes (OR 1.10, 95\% Cl 1.04-1.15), attended meetings in the community (OR 1.22, 95\% Cl 1.16-1.28), participated in hobbies or sports activities (OR 1.09, 95\% Cl 1.03-1.16), and engaged in fieldwork or gardening (OR 1.14, 95\% Cl 1.08-1.21). The imputed sample showed that non-reverters were more likely to discontinue fieldwork or gardening (11.0\% vs. 6.1\%) than reverters during the follow-up period.

Conclusions: Specific lifestyle activities may play important roles in $\mathrm{MCl}$ reversion in older adults. The longitudinal data indicate that it is reasonable to recommend that individuals continue to engage in fieldwork or gardening to increase their chance of recovery from $\mathrm{MCl}$.
\end{abstract}

Keywords: Mild cognitive impairment, Lifestyle, Activities, Risk, Dementia

\section{Background}

Mild cognitive impairment (MCI) is considered to be an intermediate state between normal cognitive aging and early dementia [1]. Identifying individuals with MCI can facilitate the timely detection of dementia and may uncover useful information regarding targets for prevention in the community [2]. However, the significance of an MCI categorization is uncertain given the longitudinal data suggesting that MCI may be unstable, as many

\footnotetext{
* Correspondence: shimada@ncgg.go.jp

${ }^{1}$ Department of Preventive Gerontology, Center for Gerontology and Social Science, National Center for Geriatrics and Gerontology, 7-430 Morioka-cho, Obu City, Aichi Prefecture 474-8511, Japan

Full list of author information is available at the end of the article
}

individuals are found to be cognitively normal in follow-up assessments. A meta-analysis that assessed reversion rates in 25 studies indicated an overall reversion rate of approximately $24 \%$ [3]. Subject-based factors include recovery from illness, differing measurement, and diagnosis methodologies as well as variations in the cutoff scores used to diagnose MCI may also increase the probability of reversion from $\mathrm{MCI}$ to normal cognition [4-6].

Although a number of previous studies have suggested that MCI is unstable, this is especially evident from studies of MCI patients presenting to memory disorder clinics, in whom the annual rate of progression to dementia is reported to range from 10 to $15 \%$ [7]. While rates of progression have been found to be lower in 
population-based studies, i.e., between 6 and 10\%, these values are higher than the 1 to $2 \%$ annualized incidence rates of dementia in the general older population [8]. Thus, we believe that recovery from $\mathrm{MCI}$ to normal cognition has important implications for the prevention of dementia.

The reduction of risk factors and promotion of protective factors is essential to the formulation of effective interventions for preventing dementia. Prospective observational studies have suggested a number of common factors related to $\mathrm{MCI}$ reversion. These include younger age, being unmarried, APOE $\varepsilon 4$ status, neuroimaging factors (i.e., larger hippocampal volumes), better cognitive function, MCI subtype, absence of an informant-based memory complaint, arthritis, high blood pressure and lipid abnormality, high mental activity, openness to experience, better vision, and better smelling ability [9-13]. Although previous studies have revealed many factors related to MCI reversion, information about reversible factors related $\mathrm{MCI}$ reversion is limited, impeding the development of intervention strategies.

The aim of the present study was to examine whether reversible factors such as lifestyle activities are associated with $\mathrm{MCI}$ reversion in elderly individuals using the $\mathrm{Na}$ tional Center for Geriatrics and Gerontology-Study of Geriatric Syndromes (NCGG-SGS) database [14]. Previous studies have suggested that elderly persons who had participated to a greater extent in everyday activities had a lower risk of dementia compared with those who had participated to a lesser extent [15-23]. Although these studies identified the efficacy of leisure-time activities including physical, cognitive, and social activities, the relationships between dementia incidence and lifestyle activities, such as visiting friends and house cleaning, are not well known. We hypothesized that participants who completed certain everyday activities would have a higher proportion of MCI reversion compared with those who had not participated in such activities.

\section{Methods}

\section{Participants}

Participants were selected from adults enrolled in a population-based cohort study titled "The Obu Study of Health Promotion for the Elderly (OSHPE)" [24], which is part of the NCGG-SGS [14]. In the present study, we analyzed longitudinal data from 396 community-dwelling older adults who were $\geq 65$ years old (mean age $71.1 \pm 4.5$ years, 181 men and 215 women), had participated in both the first and second waves of the OSHPE, and did not have $\mathrm{MCI}$ at the time of the first wave assessment. The first wave of the OSHPE was held between August 2011 and February 2012. During this wave, 5104 communitydwelling elderly people participated in a baseline OSHPE assessment. Of these, 3095 (60.6\%) took part in a second-wave cognitive examination between August 2015 and August 2016.

The inclusion criteria were residence in Obu and aged $\geq 65$ years at the time of the first examination (August 2011 to March 2013). The baseline exclusion criteria were health problems such as Alzheimer's disease, Parkinson's disease, depression, or stroke $(n=549)$; inability to perform basic tasks of daily living such as eating, grooming, bathing, and climbing up and down stairs $(n=26)$; need for support or care as certified by the Japanese public long-term care insurance system due to disability $(n=69)$; missing data regarding the exclusion criteria $(n=14)$; inability to complete cognitive tests at the baseline assessment $(n=143)$; relocation $(n=38)$ or death $(n=$ 112) during the follow-up period; normal cognition $(n=2892)$; and global cognitive decline at the baseline assessment $(n=518)$. Of the 743 potential participants, 347 did not receive the second-wave cognitive examination (Fig. 1). Thus, we analyzed data from 396 participants. Multiple imputation was used to adjust for selection bias and loss of information, because we found the potential bias of the sample in baseline data on those who remained versus left the follow-up (Additional file 1). We imputed reversion status, which was divided into reverters, who recovered from MCI to normal cognition, and non-reverters, who had $\mathrm{MCI}$, global cognitive decline (as indicated by a Mini-Mental State Examination (MMSE) [25] score of <24) and/or Alzheimer's disease (AD), for participants with missing data. We generated 50 imputed values for each participant with missing data using a fully conditional specification multiple imputation procedure assuming that those values were missing at random [26]. The fully conditional specification procedure first substituted missing data of potential predictors by plausible values using an iterative stochastic algorithm, which results in 50 multiple complemented replications of the original dataset. The main advantages of using multiple imputation over the complete sample were as follows: (1) increased power to detect associations in a multiple regression model using the partial information available for some participants and (2) this enabled us to account for the likely possibility that the presence of missing scores was not completely random, but that, among participants with similar known characteristics, the distribution of missing values would resemble that of known values [10]. All participants gave their informed consent before they were included in the study. The study protocol was approved by an institutional review board.

\section{Measurements of lifestyle activity}

Participants completed a questionnaire that comprised 16 questions regarding instrumental activities of daily living (IADL), cognitive activity, social activity, and productive 


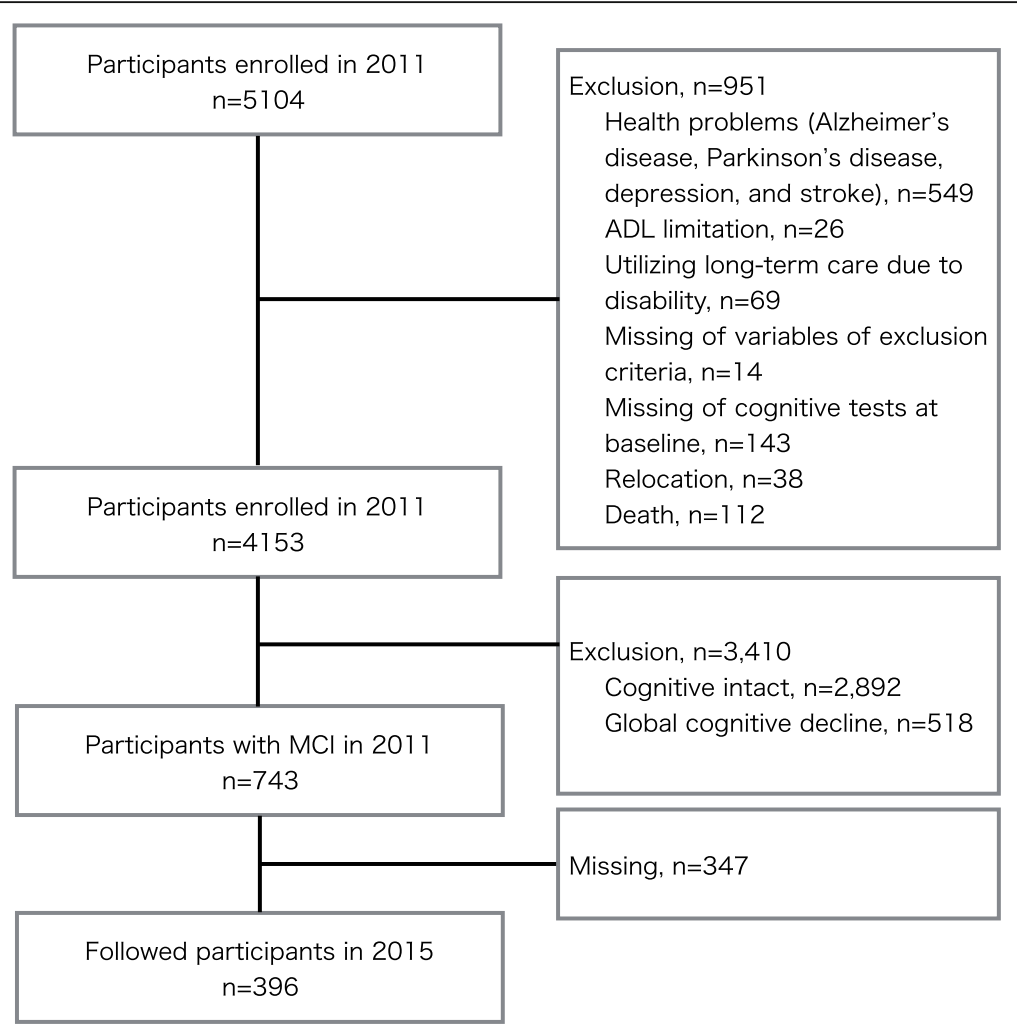

Fig. 1 Flow diagram of sample selection

activity as different elements of lifestyle activity. The questionnaire included the following questions that measured IADL: (1) "Do you go outdoors using the bus and train?," (2) "Do you engage in cash handling and banking?," (3) "Do you drive a car?," and (4) "Do you use maps to go to unfamiliar places?" Items that measured cognitive activity included (5) "Do you read books or newspapers?," (6) "Do you engage in cognitive stimulation such as board games and learning?" (7) "Do you engage in cultural classes?," and (8) "Do you use a personal computer?." Items that measured social activity included (9) "Do you talk with other people every day?," (10) "Are you sometimes called on for advice?," (11) "Do you attend meetings in the community?" and (12) "Do you engage in hobbies or sports activities?." Items that measured productive activity included (13) "Do you engage in housecleaning?," (14) "Do you engage in fieldwork or gardening?," (15) "Do you take care of a grandchild or pet?", and (16) "Do you engage in paid work?." Answers of "yes" were determined to be positive responses.

\section{Measurement of cognitive functions and incident AD}

We used the National Center for Geriatrics and Gerontology-Functional Assessment Tool (NCGG-FAT), which is an iPad application, to conduct cognitive screening [27]. The NCGG-FAT includes the following domains: (1) memory (wordlist memory-I [immediate recognition] and word list memory-II [delayed recall]); (2) attention (a tablet version of the Trail Making Test-part A; TMT-part A); (3) executive function (a tablet version of the TMT-part B), and (4) processing speed (a tablet version of the Digit Symbol Substitution Test). The NCGG-FAT has been shown to have high test-retest reliability and moderate to high criterion-related validity [28] and predictive validity [29] among community-dwelling older persons. Well-trained study assistants conducted the assessments of cognitive functioning. Before the study began, all staff received training from the authors regarding the protocols for administering the assessments. Potential participants with MCI were identified after reviewing available clinical, neuropsychological, and laboratory data at meetings involving study neurologists and neuropsychologists, as previously described [24]. In brief, the MCI participants were independently recruited using the NCGG-FAT, which has two memory tasks, tests of attention and executive function, and a processing speed task. Using established criteria [1], we diagnosed MCI in individuals who exhibited cognitive impairment but were functionally independent in terms of basic daily life activities. For all cognitive tests, we used established standardized thresholds in each corresponding domain for defining impairment in population-based cohorts 
comprising community-dwelling older persons (scores $>1.5$ standard deviations [SDs] below the age- and educationspecific means). We used the MMSE to measure global cognitive function [25]. Specifically, we used $<24$ points on the MMSE as a cutoff score for global cognitive impairment (GCI), in accordance with previous findings [30]. Participants whose scores were all $>1.5 \mathrm{SD}$ units above the mean were classified as belonging to the normal cognition (NC) group.

In the present study, participants were tracked monthly for newly incident AD, as recorded by the Japanese National Health Insurance and Later-Stage Medical Care systems [31]. Participants were considered to have $\mathrm{AD}$ if they had been diagnosed by a medical doctor according to the International Classification of Diseases, 10th revision. Incidence of AD was based simply on diagnoses by third-party doctors who were blinded to the design and participant groups of the study. Participants were divided into the following two groups: the reverter group, who had $\mathrm{CN}$ at the follow-up, and the non-reverter group, who had $\mathrm{MCI}, \mathrm{GCI}$, and/or $\mathrm{AD}$ at the follow-up.

\section{Potential confounding factors}

Demographic variables, chronic medical conditions, psychosocial factors, and physical performance are associated with cognitive decline in older persons [32, 33]. All multivariate models included the following covariates unless otherwise specified: age at enrollment, sex, educational level, current smoking status, chronic medical illnesses, depressive symptoms, and comfortable walking speed. The presence of the following self-reported chronic medical illnesses was entered into the models: heart disease, pulmonary disease, hypertension, and diabetes mellitus. Depressive symptoms were measured using the 15-item Geriatric Depression Scale (GDS) [34]. We calculated average walking speed by asking the participants to complete five trials in which they walked at a comfortable walking speed.

\section{Statistical analysis}

We used Student's $t$ tests and Pearson's chi-squared test to examine differences in the baseline participant characteristics and between the reverter and non-reverter groups. We used the chi-squared test with adjusted standardized residuals to determine whether MCI status significantly affected reversion. Residuals followed the $t$ distribution: $t>1.96$ was accepted as indicating $P<.05$, and $t>2.56$ as indicating $P<.01$.

We calculated the reversion rates from $\mathrm{MCI}$ to $\mathrm{NC}$ and non-reversion rates during the follow-up assessments. Logistic regression models were used to analyze associations between lifestyle activity status and reversion from MCI to NC. We used a multiple adjustment model that was adjusted for demographic variables, primary diseases, lifestyle, and psychological variables as possible confounding factors in the participants with complete and imputed data sets. Adjusted odds ratios (ORs) for reversion and their 95\% confidence intervals (CIs) were estimated. All analyses were performed using IBM SPSS v.25.0 (IBM Japan, Tokyo). Statistical significance was set at $P<.05$.

\section{Results}

Baseline characteristics of the participants

In the initial group of 396 participants, 202 participants (51.0\%) reverted to NC from MCI. The reversion rate for participants for whom we imputed samples was $34.3 \%$. Table 1 presents the measurements for participants with and without imputed samples. The two groups had the same mean scores and proportions except for one item regarding cognitive activity. Table 2 shows the participant baseline characteristics for those grouped according to the presence or absence of reversion from $\mathrm{MCI}$ to $\mathrm{NC}$ in the imputed group. Age, sex, educational level, smoking status, heart disease, pulmonary disease, hypertension, diabetes, walking speed, MMSE, GDS score, MCI status, and lifestyle activities differed significantly between participants with and without reversion (Table 2).

\section{Associations between reversion and lifestyle activity}

Table 3 shows the rate of reversion in participants who engaged in specific lifestyle activities. We found no significant relationships between reversion and lifestyle activities in the participants with complete data sets. In the participants for whom we imputed data, a logistic regression model showed that the odds ratios of reversion were significantly higher in participants who drove a car (OR $1.50,95 \%$ CI $1.41-1.60, P<.01)$, used maps to travel to unfamiliar places (OR 1.12, 95\% CI 1.06-1.18, $P<.01$ ), read books or newspapers (OR 1.54, 95\% CI 1.37-1.73, $P \leq .01$ ), participated in cultural classes (OR 1.10, 95\% CI 1.04-1.15, $P \leq .01$ ), attended meetings in the community (OR 1.22, 95\% CI 1.16-1.28, $P \leq .01)$, participated in hobbies or sports activities (OR 1.09, 95\% CI 1.03-1.16, $P \leq .01$ ), and engaged in fieldwork or gardening (OR 1.14, 95\% CI 1.08$1.21, P \leq .01)$. The logistic models showed negative relationships between reversion and lifestyle activities that included cognitive stimulation, such as board games and learning (OR $0.92,95 \% \mathrm{CI} 0.88-0.97, P \leq .01$ ), daily conversations (OR $0.73,95 \%$ CI $0.64-0.82, P \leq .01$ ), giving someone a helping hand (OR 0.76, 95\% CI 0.70-0.83, $P \leq .01$ ), and housecleaning (OR 0.90, 95\% CI 0.84-0.98, $P=.01$ ).

\section{Associations between reversion and longitudinal change}

Table 4 shows changes in activity patterns from the baseline to follow-up assessments. The proportion of non-reverters who started reading books or newspapers 
Table 1 Baseline characteristics in participants with complete data sets and participants for whom we imputed data

\begin{tabular}{|c|c|c|}
\hline & $\begin{array}{l}\text { Participants with } \\
\text { complete data } \\
(n=743)\end{array}$ & $\begin{array}{l}\text { Participants with } \\
\text { imputed data } \\
(n=37,893)\end{array}$ \\
\hline Age (years)* & $71.8(5.2)$ & $71.8(5.2)$ \\
\hline Sex (\% male) & 53.8 & 53.8 \\
\hline Education (years)* & $11.3(2.5)$ & $11.3(2.5)$ \\
\hline Current smoking (\% yes) & 10.6 & 10.6 \\
\hline Heart disease (\% yes) & 17.5 & 17.5 \\
\hline Pulmonary disease (\% yes) & 8.7 & 8.7 \\
\hline Hypertension (\% yes) & 47.5 & 47.5 \\
\hline Diabetes mellitus (\% yes) & 15.1 & 15.1 \\
\hline Walking speed $(\mathrm{m} / \mathrm{s})^{*}$ & $1.2(0.2)$ & $1.2(0.2)$ \\
\hline $\begin{array}{l}\text { Mini-mental state examination } \\
\text { (points)* }\end{array}$ & $26.5(1.8)$ & $26.5(1.8)$ \\
\hline Geriatric depression scale (points)* & $3.1(2.6)$ & $3.1(2.6)$ \\
\hline \multicolumn{3}{|l|}{ Category of $\mathrm{MCl}$ (\% yes) } \\
\hline Amnestic $\mathrm{MCl}$ single domain & 14.3 & 14.3 \\
\hline Non-amnestic MCl single domain & 62.2 & 62.2 \\
\hline Amnestic MCI multiple domain & 6.7 & 6.7 \\
\hline $\begin{array}{l}\text { Non-amnestic MCl multiple } \\
\text { domain }\end{array}$ & 16.8 & 16.8 \\
\hline \multicolumn{3}{|c|}{ Instrumental activities of daily living (\% yes) } \\
\hline $\begin{array}{l}\text { Going outdoors using bus and } \\
\text { train }\end{array}$ & 89.3 & 89.3 \\
\hline Cash handling and banking & 88.6 & 88.6 \\
\hline Driving a car & 67.5 & 67.5 \\
\hline $\begin{array}{l}\text { Using map to go unfamiliar } \\
\text { place }\end{array}$ & 57.9 & 57.9 \\
\hline \multicolumn{3}{|l|}{ Cognitive activity (\% yes) } \\
\hline Reading of book or newspaper & 94.1 & 94.1 \\
\hline $\begin{array}{l}\text { Cognitive stimulation such as } \\
\text { board game and learning }\end{array}$ & 44.2 & 44.2 \\
\hline Culture lesson & 39.4 & 39.4 \\
\hline Using personal computer & 28 & 26.8 \\
\hline \multicolumn{3}{|l|}{ Social activity (\% yes) } \\
\hline Daily conversation & 95.4 & 95.4 \\
\hline Giving someone a helping hand & 89.7 & 89.7 \\
\hline $\begin{array}{l}\text { Attending a meeting in the } \\
\text { community }\end{array}$ & 52.4 & 52.4 \\
\hline Hobby or sports activity & 69.4 & 69.4 \\
\hline \multicolumn{3}{|l|}{ Productive activity (\% yes) } \\
\hline Housecleaning & 87.7 & 87.7 \\
\hline Field work or gardening & 72.9 & 72.9 \\
\hline Taking care of grandchild or pet & 54.4 & 54.4 \\
\hline Working & 30.4 & 30.4 \\
\hline
\end{tabular}

*Mean (standard deviation)
Table 2 Comparison of baseline characteristics between reverters and non-reverters

\begin{tabular}{|c|c|c|c|}
\hline & $\begin{array}{l}\text { Reverters } \\
(n=12,897)\end{array}$ & $\begin{array}{l}\text { Non-reverters } \\
(n=24,649)\end{array}$ & $P$ value \\
\hline Age (years) & $69.6(3.6)$ & $73.0(5.6)$ & $<0.01$ \\
\hline Sex (\% male) & 55.6 & 52.9 & $<0.01$ \\
\hline Education (years) & $11.7(2.4)$ & $11.0(2.5)$ & $<0.01$ \\
\hline Current smoking (\% yes) & 9 & 11.4 & $<0.01$ \\
\hline Heart disease (\% yes) & 18.3 & 17.1 & $<0.01$ \\
\hline Pulmonary disease (\% yes) & 8.1 & 9.1 & $<0.01$ \\
\hline Hypertension (\% yes) & 43.4 & 49.6 & $<0.01$ \\
\hline Diabetes mellitus (\% yes) & 13.8 & 15.8 & $<0.01$ \\
\hline Walking speed (m/s) & $1.3(0.2)$ & $1.2(0.2)$ & $<0.01$ \\
\hline $\begin{array}{l}\text { Mini-mental state examination } \\
\text { (points) }\end{array}$ & $26.9(1.8)$ & $26.3(1.8)$ & $<0.01$ \\
\hline Geriatric depression scale (points) & $2.7(2.4)$ & $3.3(2.7)$ & $<0.01$ \\
\hline Category of $\mathrm{MCl}$ & & & $<0.01$ \\
\hline Amnestic $\mathrm{MCl}$ single domain & 16.3 & 13.2 & $<0.01$ \\
\hline $\begin{array}{l}\text { Non-amnestic MCl single } \\
\text { domain }\end{array}$ & 71.2 & 57.5 & $<0.01$ \\
\hline Amnestic MCI multiple domain & 5.3 & 7.5 & $<0.01$ \\
\hline $\begin{array}{l}\text { Non-amnestic MCI multiple } \\
\text { domain }\end{array}$ & 7.2 & 21.8 & $<0.01$ \\
\hline \multicolumn{4}{|c|}{ Instrumental activities of daily living (\% yes) } \\
\hline $\begin{array}{l}\text { Going outdoors using bus and } \\
\text { train }\end{array}$ & 90.4 & 88.8 & $<0.01$ \\
\hline Cash handling and banking & 89.1 & 88.4 & 0.08 \\
\hline Driving a car & 76.8 & 62.7 & $<0.01$ \\
\hline $\begin{array}{l}\text { Using map to go unfamiliar } \\
\text { place }\end{array}$ & 63.7 & 54.9 & $<0.01$ \\
\hline \multicolumn{4}{|l|}{ Cognitive activity (\% yes) } \\
\hline Reading of book or newspaper & 96.5 & 92.9 & $<0.01$ \\
\hline $\begin{array}{l}\text { Cognitive stimulation such as } \\
\text { board game and learning }\end{array}$ & 46.6 & 42.9 & $<0.01$ \\
\hline Culture lesson & 43.3 & 37.4 & $<0.01$ \\
\hline Using personal computer & 33.3 & 23.4 & $<0.01$ \\
\hline \multicolumn{4}{|l|}{ Social activity (\% yes) } \\
\hline Daily conversation & 96.4 & 94.9 & $<0.01$ \\
\hline Giving someone a helping hand & 91.2 & 89 & $<0.01$ \\
\hline $\begin{array}{l}\text { Attending a meeting in the } \\
\text { community }\end{array}$ & 55.9 & 50.6 & $<0.01$ \\
\hline Hobby or sports activity & 74.5 & 66.8 & $<0.01$ \\
\hline \multicolumn{4}{|l|}{ Productive activity (\% yes) } \\
\hline Housecleaning & 88.5 & 87.3 & $<0.01$ \\
\hline Field work or gardening & 74.6 & 72 & $<0.01$ \\
\hline Taking care of grandchild or pet & 59 & 52 & $<0.01$ \\
\hline Working & 33.8 & 28.7 & $<0.01$ \\
\hline
\end{tabular}


Table 3 Odds ratios for mild cognitive impairment reversion according to lifestyle activity status (yes/no)

\begin{tabular}{|c|c|c|c|c|}
\hline & \multicolumn{2}{|c|}{ Participants with complete data } & \multicolumn{2}{|c|}{ Participants with imputed data } \\
\hline & Odds ratio $(95 \% \mathrm{Cl})$ & $P$ value & Odds ratio $(95 \% \mathrm{Cl})$ & $P$ value \\
\hline \multicolumn{5}{|l|}{ Instrumental activities of daily living (\% yes) } \\
\hline Going outdoors using bus and train & $0.59(0.25-1.41)$ & 0.24 & $0.95(0.87-1.02)$ & 0.17 \\
\hline Cash handling and banking & $1.04(0.49-2.23)$ & 0.92 & $0.97(0.89-1.05)$ & 0.39 \\
\hline Driving a car & $1.65(0.9-3.05)$ & 0.11 & $1.50(1.41-1.60)$ & $<0.01$ \\
\hline Using map to go unfamiliar place & $0.89(0.52-1.52)$ & 0.68 & $1.12(1.06-1.18)$ & $<0.01$ \\
\hline \multicolumn{5}{|l|}{ Cognitive activity (\% yes) } \\
\hline Reading of book or newspaper & $1.73(0.43-6.98)$ & 0.44 & $1.54(1.37-1.73)$ & $<0.01$ \\
\hline Cognitive stimulation such as board game and learning & $0.97(0.59-1.6)$ & 0.91 & $0.92(0.88-0.97)$ & $<0.01$ \\
\hline Culture lesson & $0.83(0.5-1.38)$ & 0.47 & $1.10(1.04-1.15)$ & $<0.01$ \\
\hline Using personal computer & $1(0.57-1.75)$ & 0.99 & $1.03(0.97-1.09)$ & 0.31 \\
\hline \multicolumn{5}{|l|}{ Social activity (\% yes) } \\
\hline Daily conversation & $0.54(0.16-1.9)$ & 0.34 & $0.73(0.64-0.82)$ & $<0.01$ \\
\hline Giving someone a helping hand & $0.53(0.22-1.29)$ & 0.16 & $0.76(0.70-0.83)$ & $<0.01$ \\
\hline Attending a meeting in the community & $1.05(0.64-1.73)$ & 0.84 & $1.22(1.16-1.28)$ & $<0.01$ \\
\hline Hobby or sports activity & $0.84(0.47-1.47)$ & 0.53 & $1.09(1.03-1.16)$ & $<0.01$ \\
\hline \multicolumn{5}{|l|}{ Productive activity (\% yes) } \\
\hline Housecleaning & $0.98(0.46-2.09)$ & 0.96 & $0.90(0.84-0.98)$ & 0.01 \\
\hline Field work or gardening & $1.07(0.61-1.86)$ & 0.82 & $1.14(1.08-1.21)$ & $<0.01$ \\
\hline Taking care of grandchild or pet & $1.11(0.69-1.79)$ & 0.66 & $1.01(0.96-1.06)$ & 0.64 \\
\hline Working & $0.91(0.55-1.49)$ & 0.70 & $1.02(0.97-1.07)$ & 0.55 \\
\hline
\end{tabular}

and stopped fieldwork or gardening was significantly higher than that of reverters during the 4-year follow-up period among the participants with complete data. The imputed sample showed that the proportion of non-reverters who began to drive a car, read books or newspapers, attended a meeting, or engaged in hobbies or sports increased significantly compared with reverters during the 4-year follow-up. However, non-reverters were significantly more likely to discontinue driving a car, read books or newspapers, and engage in hobbies or sports compared with reverters. The proportion of reverters who discontinued using maps, participation in cultural lessons, and attendance to meetings increased significantly compared with non-reverters in the imputed sample (Table 4).

\section{Discussion}

Although previous studies have reported many factors related to MCI reversion [9-13], information regarding reversible factors related to MCI reversion is limited. In this prospective study, reversion from MCI to NC was significantly associated with specific lifestyle activities after adjusting logistic regression models for many potential confounders. Participants were more likely to revert if they started driving a car, used maps to travel to unfamiliar places, read books or newspapers, participated in cultural lessons, attended community meetings, carried out hobbies or sports, and engaged in fieldwork or gardening among the participants with imputed data. Driving cessation is associated with a number of negative consequences, such as declined general health [35], cognitive decline [36], depressive symptoms [37], increased risk for long-term care institutionalization [38], and mortality [39]. The results suggest that driving is associated with maintenance of cognitive function. Additionally, increasing the amount of time spent driving may promote the use of maps, which is likely beneficial for improving cognitive function. In terms of cognitive activity, our work is consistent with previous findings indicating that participation in cognitive activities is associated with reduced rates of cognitive decline in elderly people $[40,41]$. However, we found that specific cognitive activities, such as reading books or newspapers, were particularly associated with reversion from MCI to NC. Low levels of social and IADL activities are considered to predict future functional decline among community-dwelling older people [42], and human interaction has a positive influence on mental health [43]. Meetings and hobbies or sports are social activities that include human interaction. Thus, it is unsurprising that these activities are related to MCI reversion. In terms of productive activities, countrywide and population-based research has indicated that gardening may be beneficial for adult mental health, 
Table 4 Changes in lifestyle activities between baseline and follow-up assessments

\begin{tabular}{|c|c|c|c|c|c|c|}
\hline & Participant & omplete data & & Participant & imputed data & \\
\hline & Reverters & Non-reverters & $P$ value & Reverters & Non-reverters & $P$ value \\
\hline Driving a car & & & 0.50 & & & $<.01$ \\
\hline Unchanged & 97.3 & 95.3 & $>.05$ & 97.3 & 95.6 & $<.01$ \\
\hline Begun & 1.6 & 3.6 & $>.05$ & 1.6 & 2.7 & $<.01$ \\
\hline Discontinued & 1.1 & 1.2 & $>.05$ & 1.1 & 1.7 & $<.01$ \\
\hline Using map to go unfamiliar place & & & .99 & & & $<.01$ \\
\hline Unchanged & 75.4 & 76.2 & $>.05$ & 76.4 & 77.8 & $<.01$ \\
\hline Begun & 8 & 7.7 & $>.05$ & 8.1 & 8.1 & $>.05$ \\
\hline Discontinued & 16.6 & 16.1 & $>.05$ & 15.5 & 14.1 & $<.01$ \\
\hline Reading of book or newspaper & & & .01 & & & $<.01$ \\
\hline Unchanged & 98.9 & 92.9 & $<.01$ & 98.4 & 93.0 & $<.01$ \\
\hline Begun & 0 & 3.0 & $<.05$ & 0.3 & 2.9 & $<.01$ \\
\hline Discontinued & 1.1 & 4.1 & $>.05$ & 1.3 & 4.1 & $<.01$ \\
\hline Culture lesson & & & .82 & & & $<.01$ \\
\hline Unchanged & 83.3 & 83.8 & $>.05$ & 84.5 & 87.3 & $<.01$ \\
\hline Begun & 4.3 & 5.4 & $>.05$ & 3.9 & 3.7 & $>.05$ \\
\hline Discontinued & 12.4 & 10.8 & $>.05$ & 11.7 & 9.0 & $<.01$ \\
\hline Attending a meeting in the community & & & .63 & & & $<.01$ \\
\hline Unchanged & 74.2 & 78.4 & $>.05$ & 74.9 & 76.7 & $<.01$ \\
\hline Begun & 10.2 & 9.0 & $>.05$ & 10.4 & 11.4 & $<.01$ \\
\hline Discontinued & 15.6 & 12.6 & $>.05$ & 14.7 & 11.8 & $<.01$ \\
\hline Hobby or sports activity & & & .54 & & & $<.01$ \\
\hline Unchanged & 81.3 & 76.5 & $>.05$ & 81.5 & 77.8 & $<.01$ \\
\hline Begun & 7.5 & 14.5 & $>.05$ & 7.2 & 8.7 & $<.01$ \\
\hline Discontinued & 11.2 & 9.0 & $>.05$ & 11.3 & 13.5 & $<.01$ \\
\hline Field work or gardening & & & .03 & & & $<.01$ \\
\hline Unchanged & 86.6 & 77.7 & $<.05$ & 85.9 & 81.5 & $<.01$ \\
\hline Begun & 8.1 & 9.0 & $>.05$ & 8.0 & 7.5 & $>.05$ \\
\hline Discontinued & 5.4 & 13.3 & $<.01$ & 6.1 & 11.0 & $<.01$ \\
\hline
\end{tabular}

including self-rated health and psychological distress [44]. Our findings regarding the relationship between gardening or fieldwork and MCI reversion in older populations support these previous results. Although some lifestyle activities were associated positively with reversion from $\mathrm{MCI}$ to NC, board games and learning, daily conversation, giving someone a helping hand, and housecleaning were negatively correlated with MCI reversion. The implementation rates of these activities (with the exception of board games) were high, about $90 \%$, which may have led to inconsistent results with respect to previous studies.

Regarding changes in lifestyle activities between baseline and follow-up assessments, more non-reverters discontinued driving (1.7\% vs. $1.1 \%)$, reading books or newspapers $(4.1 \%$ vs. $1.3 \%)$, and participation in hobbies or sports activities (13.5\% vs. $11.3 \%)$ during the study period, while more non-reverters increased engagement in these activities. Thus, the evidence is not sufficiently persuasive to recommend initiation of these activities or an increase in participation for the purpose of preventing decline or reversing MCI. However, more non-reverters discontinued fieldwork or gardening $(11.0 \%$ vs. $6.1 \%)$ during the study period and non-reverters did not increase engagement in these activities. Thus, it is reasonable to recommend continuation of fieldwork or gardening for recovery from MCI.

This study had several limitations. Previous research using cluster analytic techniques has demonstrated that MCI participants can be grouped based on similarities in their neuropsychological profiles, providing a method of describing MCI subtypes without being confined to the amnestic/non-amnestic distinction $[45,46]$. One critical finding from a cluster analytic study was the identification of a large subgroup who performed within normal 
limits on cognitive testing despite their $\mathrm{MCI}$ diagnosis [45]. The cluster-derived normal group comprised one third (34\%) of the Alzheimer's Disease Neuroimaging Initiative MCI sample and was comparable to a robust normal control group in cognitive test performance and percentage of individuals with positive cerebrospinal fluid biomarkers for $\mathrm{AD}$ [47]. In addition, the cluster-derived normal group had fewer APOE- $\varepsilon 4$ carriers and fewer individuals with positive CSF biomarkers of $\mathrm{AD}$ than the $\mathrm{MCI}$ groups and was also less likely to progress to $\mathrm{AD}$ and more likely to revert to normal than the MCI groups [47]. These results suggest that the conventional diagnosis of MCI may be highly susceptible to false positive diagnostic errors, which is consistent with previous reports of high reversion rates or lack of progression in those with $\mathrm{MCI}$ [3]. The high reversion rate in the study may be affected by the false positive issue, and it is difficult to accurately determine the influence of activities on reversion MCI to NC. And the participants were not randomly recruited, and about $47 \%$ of the participants dropped out at the follow-up. This dropout rate may have led to underrepresentation of cognitive decline and dementia. Moreover, we did not collect data about potential correlates, such as nutritional status, which could affect MCI reversion.

One strength of the present study is that our findings are consistent with comprehensive geriatric assessments designed to collect information about lifestyle activity. In addition, to our knowledge, this is the first study to use a large population-based sample to examine correlations between specific lifestyle activities and MCI reversion.

\section{Conclusions}

The present results indicate that reversion from $\mathrm{MCI}$ to $\mathrm{NC}$ was significantly associated with specific lifestyle activities including driving a car, using maps to travel to unfamiliar places, reading books or newspapers, taking culture lessons, attending community meetings, participating in hobbies or sports activities, and doing fieldwork or gardening among the participants with imputed samples. The longitudinal data indicate that continuing fieldwork or gardening may increase the chance of recovery from MCI.

\section{Additional file}

Additional file 1: Baseline characteristics in participants who remained versus left the follow-up. (DOCX $18 \mathrm{~kb}$ )

\section{Abbreviations}

AD: Alzheimer's disease; Cl: Confidence interval; GCl: global cognitive impairment; GDS: Geriatric Depression Scale; IADL: Instrumental activities of daily living; MCl: Mild cognitive impairment; MMSE: Mini-Mental State Examination; NC: Normal cognition; NCGG-FAT: National Center for Geriatrics and Gerontology-Functional Assessment Tool; NCGG-SGS: National Center for Geriatrics and Gerontology - Study of Geriatric Syndromes; OR: Odds ratio;
OSHPE: Obu Study of Health Promotion for the Elderly; SD: Standard deviation; TMT: Trail Making Test

\section{Acknowledgements}

We wish to thank the health care staff for their assistance with the assessments.

\section{Funding}

This work was supported by a Health and Labour Sciences Research Grant (Comprehensive Research on Aging and Health), the Research Institute of Science and Technology for Society (RISTEX) from the Japan Science and Technology Agency (JST) for redesigning communities for an aged society in 2012, a Grant-in-Aid for Scientific Research (B) (grant number 23300205), and Research Funding for Longevity Sciences (22-16) from the National Center for Geriatrics and Gerontology, Japan. No support was received from sources associated with industry.

Availability of data and materials

The datasets used and/or analyzed during the present study are available from the corresponding author on reasonable request.

\section{Authors' contributions}

HS planned the study, wrote the first draft of the manuscript, and coordinated the review and editing process leading to the final manuscript HM participated in the design of the study and wrote the paper. SL and TD collected the data and contributed to the editorial process and review of the manuscript. All authors carried out critical revision of the manuscript for important intellectual content. All authors read and approved the final manuscript.

\section{Competing interest}

The authors declare that they have no competing interests.

\section{Ethics approval and consent to participate}

All participants gave their informed consent before they were included in the study. The study protocol was approved by the Ethics Committee of the National Center for Geriatrics and Gerontology.

Consent for publication

Not applicable.

\section{Publisher's Note}

Springer Nature remains neutral with regard to jurisdictional claims in published maps and institutional affiliations.

\section{Author details}

1Department of Preventive Gerontology, Center for Gerontology and Social Science, National Center for Geriatrics and Gerontology, 7-430 Morioka-cho, Obu City, Aichi Prefecture 474-8511, Japan. ${ }^{2}$ Department of Physical Therapy, School of Health Sciences, Faculty of Medicine, Kagoshima University, Kagoshima City, Japan.

Received: 12 September 2018 Accepted: 28 February 2019

Published online: 13 March 2019

\section{References}

1. Petersen RC. Mild cognitive impairment as a diagnostic entity. J Intern Med. 2004;256(3):183-94.

2. Aerts $L$, Heffernan $M$, Kochan NA, et al. Effects of $\mathrm{MCl}$ subtype and reversion on progression to dementia in a community sample. Neurol. 2017:88(23): 2225-32.

3. Malek-Ahmadi M. Reversion from mild cognitive impairment to normal cognition: a meta-analysis. Alzheimer Dis Assoc Disord. 2016;30(4):324-30.

4. Petersen RC, Caracciolo B, Brayne C, Gauthier S, Jelic V, Fratiglioni L. Mild cognitive impairment: a concept in evolution. J Intern Med. 2014;275(3): 214-28.

5. Cook SE, Marsiske M, Thomas KR, et al. Identification of mild cognitive impairment in ACTIVE: algorithmic classification and stability. J Int Neuropsychol Soc. 2013;19(1):73-87. 
6. de Jager CA, Budge MM. Stability and predictability of the classification of mild cognitive impairment as assessed by episodic memory test performance over time. Neurocase. 2005;11(1):72-9.

7. Farias ST, Mungas D, Reed BR, Harvey D, DeCarli C. Progression of mild cognitive impairment to dementia in clinic- vs community-based cohorts. Arch Neurol. 2009;66(9):1151-7.

8. Petersen RC, Smith GE, Waring SC, Ivnik RJ, Tangalos EG, Kokmen E. Mild cognitive impairment: clinical characterization and outcome. Arch Neurol. 1999:56(3):303-8.

9. Sachdev PS, Lipnicki DM, Crawford J, et al. Factors predicting reversion from mild cognitive impairment to normal cognitive functioning: a populationbased study. PLoS One. 2013;8(3):e59649.

10. Koepsell TD, Monsell SE. Reversion from mild cognitive impairment to normal or near-normal cognition: risk factors and prognosis. Neurol. 2012; 79(15):1591-8.

11. Pandya SY, Lacritz LH, Weiner MF, Deschner M, Woon FL. Predictors of reversion from mild cognitive impairment to normal cognition. Dement Geriatr Cogn Disord. 2017;43(3-4):204-14.

12. Osone A, Arai R, Hakamada R, Shimoda K. Impact of lifestyle-related disease on conversion and reversion in patients with mild cognitive impairment: after 12 months of follow-up. Int J Geriatr Psychiatry. 2016;31(7):740-8.

13. Osone A, Arai R, Hakamada R, Shimoda K. Cognitive and brain reserve in conversion and reversion in patients with mild cognitive impairment over 12 months of follow-up. J Clin Exp Neuropsychol. 2016;38(10):1084-93.

14. Shimada $\mathrm{H}$, Makizako $\mathrm{H}$, Lee $\mathrm{S}$, et al. Impact of cognitive frailty on daily activities in older persons. J Nutr Health Aging. 2016:1-7.

15. Fabrigoule C, Letenneur L, Dartigues JF, Zarrouk M, Commenges D, Barberger-Gateau P. Social and leisure activities and risk of dementia: a prospective longitudinal study. J Am Geriatr Soc. 1995;43(5):485-90.

16. Wang HX, Karp A, Winblad B, Fratiglioni L. Late-life engagement in social and leisure activities is associated with a decreased risk of dementia: a longitudinal study from the Kungsholmen project. Am J Epidemiol. 2002; 155(12):1081-7.

17. Karp A, Paillard-Borg S, Wang HX, Silverstein M, Winblad B, Fratiglioni L. Mental, physical and social components in leisure activities equally contribute to decrease dementia risk. Dement Geriatr Cogn Disord. 2006; 21(2):65-73.

18. Laurin D, Verreault R, Lindsay J, MacPherson K, Rockwood K. Physical activity and risk of cognitive impairment and dementia in elderly persons. Arch Neurol. 2001;58(3):498-504.

19. Scarmeas N, Levy G, Tang MX, Manly J, Stern Y. Influence of leisure activity on the incidence of Alzheimer's disease. Neurol. 2001;57(12):2236-42.

20. Wilson RS, Mendes De Leon CF, Barnes LL, et al. Participation in cognitively stimulating activities and risk of incident Alzheimer disease. JAMA. 2002; 287(6):742-8.

21. Wilson RS, Bennett DA, Bienias JL, et al. Cognitive activity and incident AD in a population-based sample of older persons. Neurol. 2002;59(12):1910-4

22. Sorman DE, Sundstrom A, Ronnlund M, Adolfsson R, Nilsson LG. Leisure activity in old age and risk of dementia: a 15-year prospective study. J Gerontol B Psychol Sci Soc Sci. 2014;69(4):493-501.

23. Akbaraly TN, Portet F, Fustinoni $\mathrm{S}$, et al. Leisure activities and the risk of dementia in the elderly: results from the Three-City Study. Neurol. 2009;73(11):854-61.

24. Shimada H, Makizako H, Doi T, et al. Combined prevalence of frailty and mild cognitive impairment in a population of elderly Japanese people. J Am Med Dir Assoc. 2013;14(7):518-24.

25. Folstein MF, Folstein SE, McHugh PR. "Mini-mental state". A practical method for grading the cognitive state of patients for the clinician. J Psychiatr Res. 1975;12(3):189-98.

26. van Buuren S. Multiple imputation of discrete and continuous data by fully conditional specification. Stat Methods Med Res. 2007;16(3):219-42.

27. Egan MF, Kojima M, Callicott JH, et al. The BDNF val66met polymorphism affects activity-dependent secretion of BDNF and human memory and hippocampal function. Cell. 2003;112(2):257-69.

28. Makizako $H$, Shimada $H$, Park $H$, et al. Evaluation of multidimensional neurocognitive function using a tablet personal computer: test-retest reliability and validity in community-dwelling older adults. Geriatr Gerontol Int. 2013;13(4):860-6

29. Shimada H, Makizako H, Park H, Doi T, Lee S. Validity of the National Center for Geriatrics and Gerontology-functional assessment tool and mini-mental state examination for detecting the incidence of dementia in older Japanese adults. Geriatr Gerontol Int Epub ahead of print. 2017;
30. O'Bryant SE, Humphreys JD, Smith GE, et al. Detecting dementia with the mini-mental state examination in highly educated individuals. Arch Neurol. 2008;65(7):963-7.

31. Ministry of Health $L$, and Welfare of Japan. Annual Health, Labour, and Welfare Report 2011-2012. 2012. http://www.mhlw.go.jp/english/wp/wphw6/dl/02e.pdf. Accessed 1 Jan 2017

32. Verghese J, Lipton RB, Katz MJ, et al. Leisure activities and the risk of dementia in the elderly. N Engl J Med. 2003;348(25):2508-16.

33. Morley JE, Morris JC, Berg-Weger M, et al. Brain health: the importance of recognizing cognitive impairment: an IAGG consensus conference. J Am Med Dir Assoc. 2015:16(9):731-9.

34. Yesavage JA. Geriatric depression scale. Psychopharmacol Bull. 1988;24(4): 709-11.

35. Edwards JD, Lunsman M, Perkins M, Rebok GW, Roth DL. Driving cessation and health trajectories in older adults. J Gerontol A Biol Sci Med Sci. 2009; 64(12):1290-5

36. Choi M, Lohman MC, Mezuk B. Trajectories of cognitive decline by driving mobility: evidence from the health and retirement study. Int J Geriatr Psychiatry. 2013;29(5):447-53.

37. Marottoli RA, Mendes de Leon CF, Glass TA, et al. Driving cessation and increased depressive symptoms: prospective evidence from the New Haven EPESE. Established Populations for Epidemiologic Studies of the Elderly. J Am Geriatr Soc. 1997:45(2):202-6.

38. Freeman EE, Gange SJ, Munoz B, West SK. Driving status and risk of entry into long-term care in older adults. Am J Public Health. 2006;96(7):1254-9.

39. Edwards JD, Perkins M, Ross LA, Reynolds SL. Driving status and three-year mortality among community-dwelling older adults. J Gerontol A Biol Sci Med Sci. 2009;64(2):300-5.

40. Ghisletta P, Bickel JF, Lovden M. Does activity engagement protect against cognitive decline in old age? Methodological and analytical considerations. J Gerontol B Psychol Sci Soc Sci. 2006;61(5):P253-61.

41. Hall CB, Lipton RB, Sliwinski M, Katz MJ, Derby CA, Verghese J. Cognitive activities delay onset of memory decline in persons who develop dementia. Neurol. 2009;73(5):356-61.

42. Fujiwara Y, Shinkai S, Kumagai S, et al. Longitudinal changes in higher-level functional capacity of an older population living in a Japanese urban community. Arch Gerontol Geriatr. 2003;36(2):141-53.

43. Kawachi I, Berkman LF. Social ties and mental health. J Urban Health. 2001; 78(3):458-67.

44. Shive I. Gardening is beneficial for adult mental health: Scottish Health Survey, 2012-2013. Scand J Occup Ther. 2016;23(4):320-5.

45. Clark LR, Delano-Wood L, Libon DJ, et al. Are empirically-derived subtypes of mild cognitive impairment consistent with conventional subtypes? J Int Neuropsychol Soc. 2013;19(6):635-45

46. Libon DJ, Xie SX, Eppig J, et al. The heterogeneity of mild cognitive impairment: a neuropsychological analysis. J Int Neuropsychol Soc. 2010; 16(1):84-93.

47. Edmonds EC, Delano-Wood L, Clark LR, et al. Susceptibility of the conventional criteria for mild cognitive impairment to false-positive diagnostic errors. Alzheimers Dement. 2015;11(4):415-24.

Ready to submit your research? Choose BMC and benefit from:

- fast, convenient online submission

- thorough peer review by experienced researchers in your field

- rapid publication on acceptance

- support for research data, including large and complex data types

- gold Open Access which fosters wider collaboration and increased citations

- maximum visibility for your research: over $100 \mathrm{M}$ website views per year

At $\mathrm{BMC}$, research is always in progress.

Learn more biomedcentral.com/submission 